\title{
Manejo de la intoxicación por plomo en la niñez
}

\author{
Morri E Markowitz, MC.(1)
}

\begin{abstract}
Markowitz ME.
Manejo de la intoxicación por plomo en la niñez. Salud Publica Mex 2003;45 supl 2:S225-S231.

El texto completo en inglés de este artículo está disponible en: http://www.insp.mx/salud/index.html
\end{abstract}

\section{Resumen}

El presente artículo hace una revisión del manejo clínico de la intoxicación por plomo en la niñez. Se menciona las definiciones de las diferentes técnicas de medición usadas para la determinación de plomo, y se destacan sus ventajas y posibles limitaciones. La medición de los niveles de plomo sanguíneo puede realizarse utilizando muestra de sangre antico agulada por espectrofo to metría de absorción ató mica voltametría anódica. Sin embargo, un método más eficiente para medir las concentraciones de plomo en hueso es mediante fluorescencia de Rayos X o para la determinación sistémica en un nivel bioquímico una técnica adecuada es la determinación de los niveles de plomo en orina. El tratamiento incluye la eliminación de la fuente de exposición, cambios en los hábitos de los niños y una dieta adecuada en calcio y hierro. La quelación con edetato de calcio y succimer elimina el plomo del esqueleto, el cual es eliminado por riñón; puede salvar vidas cuando la intoxicación es importante, y existe una reducción a corto plazo seguida de un aumento subsecuente de los niveles de plomo sanguíneo. En casos graves suele requerirse de dosis repetidas. La terapia de quelación puede ser necesaria en casos de niveles de plomo sanguíneo por arriba de $45 \mu \mathrm{g} / \mathrm{dl}$. La quelación reduce los niveles de plomo sanguíneo y los síntomas asociados, sin embargo, la disminución cognoscitiva puede ser irreversible, por lo que la utilización de medidas preventivas es mucho mejor que las de curación. El texto completo en inglés de este artículo está disponible en:http://www.insp.mx/ salud/index.html

Palabras clave: envenenamiento por plomo/terapia; niño

\author{
Markowitz ME. \\ Managing childhood lead poisoning. \\ Salud Publica Mex 2003;45 suppl 2:S225-S231. \\ The English version of this paper \\ is available at: http://www.insp.mx/salud/index.html
}

\begin{abstract}
A bstract
This paper reviews the clinical management of children with lead poisoning. A first step is to define the measures to be used in their assessment and be aware of the limitations. Measurements of blood lead levels can be made on anticoagulated whole blood samples using either: atomic absorption spectroscopy or anodic stripping voltametry. However a more accurate method is fluorescent RX'ray of the skeleton or systematic biochemical tests of lead levels in urine. Remedies include elimination of lead in the environment, changes in children's behavior and dietary checks for adequate calcium and iron intake. C helation therapy, using $\mathrm{C}$ a edetate and succimer eliminates lead from the skeleton, which is then quickly excleted using a cathartic to help prevent re-absorption. Chelation may save lives where BLLS are very high. There is usually a short term reduction of BLLs with a subsequent rise. Serious cases may require repeat therapies. Chelation should be considered in children with BLLs $>=45 \mu \mathrm{g} / \mathrm{dl}$. Chelation therapy reduces BLLs and associated symptoms. However cognitive decline may be irreversible, indicating that emphasis should be on prevention rather than cure. The English version of this paper is available at: http://www.insp.mx/salud/index.html
\end{abstract}

Key words: lead poisoning/ther apy; child

(1) Pediatric Environmental Sciences Clinics Children's Hospital at Montefiore.

Fecha de recibido: 1 de febrero de 2002 - Fecha de aprobado: 8 de octubre de 2002

Solicitud de sobretiros: MC. Morri Markowitz, Pediatric Environmental Sciences Clinics Children's Hospital at Montefiore, 111 E. $210^{\text {th }}$ Street, Bronx, NY 10467

Correo electrónico: markowit@ aecom.yu.edu 
E n este trabajo se hará una revisión del manejo clínico de los niños que padecen intoxicación por plomo. Con tal fin, es necesario comenzar por definir las medidas que deben aplicarse en su evaluación y tomar conciencia de sus limitaciones. El siguiente paso consiste en tomar nota de que, para que el tratamiento tenga éxito, se requiere de un enfoque multifacético y multidisciplinario.

\section{Mediciones del plomo}

Los niveles de plomo en sangre (NPS) constituyen la regla de oro para determinar el tratamiento que se debe aplicar a los niños expuestos a este metal..$^{1}$ Para fines clínicos, esta medición se lleva a cabo en una muestra de sangre completa anticoagulada, empleando cualquiera de las siguientes dos técnicas: la espectroscopía de absorción atómica, o bien, la voltimetría anódica. Más de 97\% del plomo que se llega a encontrar en una muestra de sangre está asociado con los glóbulos rojos. Por ende, esta medición refleja el contenido de plomo de un solo compartimento, esto es, el espacio vascular.

Existen limitaciones importantes en el uso de las mediciones de NPS cuando constituyen el método principal para evaluar la intoxicación por éste. Cuando los NPS son de tal magnitud que se pueden llegar a detectar, entonces constituyen un indicio de que hubo exposición, así como absorción. No obstante, los NPS tienen una correlación mínima con los síntomas y la intoxicación. No indican ni la duración ni el alcance de la exposición, y tampoco predicen, necesariamente, la cantidad que se ha absorbido. El esqueleto ${ }^{2}$ es la parte del organismo donde llega a acumularse la mayor cantidad de plomo. Las mediciones simples no proporcionan indicadores de su contenido en hueso tan claros como los que se pueden obtener in vivo mediante la metodología de la fluorescencia de rayos $X$. Las poblaciones con contenidos elevados de plomo en hueso pueden llegar a presentar NPS aceptables, i.e. $<10 \mu \mathrm{g} / \mathrm{dl}^{3}{ }^{3} \mathrm{Si}$ la detección se basa únicamente en los NPS, esas poblaciones pasarán desapercibidas.

Se han aplicado otras medidas para evaluar el grado de intoxicación por el metal. Además de su presencia en sangre y en hueso, también se encuentra en la orina. Es poca la información que se obtiene al valorar el plomo excretado espontáneamente, sin embargo, se obtiene más información a partir de los resultados de las pruebas de estimulación. En esas circunstancias, una persona que ha estado expuesta al metal recibe una dosis de alguna sustancia que se enlaza con el plomo, y posteriormente se le toma una muestra de orina (entre 6 y 8 horas más tarde) para determinar su contenido. ${ }^{4}$ Los niveles elevados de plomo en orina, resultantes de esas pruebas de reto presentan una correlación con los NPS y en hueso más elevados, y con las mediciones bioquímicas de intoxicación. ${ }^{5}$ En los adultos se asocian con exposiciones ocupacionales a largo plazo. En el sentido más literal, las pruebas de reto permiten estimar las posibilidades de éxito que tendrá un tratamiento con base en medicamentos, para poder eliminar el elemento del organismo de una persona.

La valoración bioquímica de los efectos del plomo también puede servir como guía para descubrir el grado de intoxicación. La prueba más ampliamente disponible es la medición del nivel de protoporfirina eritrocítica (PE). La protoporfirina es el sustrato de la hierroquelasa que constituye la última enzima del grupo hem. Este grupo está distribuido de manera ubicua. Por ende, los niveles de PE constituyen un indicador de los efectos del plomo en todos los tejidos. En general, existe una correlación entre estos niveles y los de la sangre cuando estos últimos superan los 20 $\mu \mathrm{g} / \mathrm{dl} .^{6} \mathrm{Si}$ bien se trata de una prueba para medir los efectos del plomo cuyo costo no es elevado, tiene, por otra parte, el inconveniente de que los niveles elevados de EP también pueden presentarse en caso de existir una deficiencia de hierro o bien alguna enfermedad inflamatoria.

¿Qué resume esta discusión? Básicamente que los NPS constituyen un punto de partida idóneo para determinar si ha ocurrido la intoxicación por el metal. Como se indica más adelante, los NPS se utilizan ampliamente para determinar la terapia que habrá de aplicarse. Sin embargo, no representan el único elemento determinante para iniciar un tratamiento o bien para conocer su carga de plomo en el organismo.

\section{Componentes del tratamiento}

El plomo en sangre tiene una vida media medida en semanas. ${ }^{7}$ Por el contrario, el del hueso tiene una vida media que se mide en años. En los niños intoxicados por plomo, cerca de los dos tercios de éste almacenados en su organismo se ubican en el esqueleto. Esa proporción aumenta a más de $90 \%$ en los adultos. ${ }^{2}$ Existe una clara correlación entre los síntomas y los NPS: los síntomas de tipo gastrointestinal son más comunes en los niños que presentan NPS > $20 \mu \mathrm{g} / \mathrm{dl}$; en aquellos con NPS próximos a los $25 \mu \mathrm{g} / \mathrm{dl}$ se observa hiperactividad, y la encefalopatía rara vez se presenta en niños con niveles NS inferiores a los $100 \mu \mathrm{g} / \mathrm{dl}$. Los objetivos de la terapia son evitar una mayor intoxicación, 
revertir este efecto, reducir el contenido de plomo en el organismo, y, en primer lugar, evitar que ocurra la intoxicación por éste.

El primer principio rector del tratamiento es la prevención. Una vez que el plomo se ha alojado en el hueso, el tratamiento para eliminarlo no es muy efectivo. Cuando se ha alojado en el cerebro, el tratamiento puede resultar demasiado tardío para ser eficaz. No obstante, puede prevenir con la progresión de los síntomas y salvar la vida.

\section{Los componentes del tratamiento}

Existen cuatro aspectos en la terapia para los niños expuestos a plomo: eliminación de la exposición ambiental; modificación de las actividades mano-boca que llevan el plomo del ambiente al organismo del niño; administración de suplementos nutricionales, en particular con aquellos metales esenciales que compiten con el plomo, y tratamiento médico para estimular la excreción.

Los primeros tres aspectos de la intervención -ambiente, conducta y nutrición- son los indicados para todos los niños que se encuentran en riesgo de exposición a plomo, pero que no necesariamente están intoxicados por dicho metal. El último aspecto, la terapia de quelación, está restringido únicamente a los casos de niños que presentan elevados NPS.

\section{Ambiente}

La erradicación de las fuentes de exposición a plomo, en una comunidad, constituye el aspecto más importante del tratamiento. Desafortunadamente las fuentes de exposición a plomo en el ambiente son innumerables. Al ser un metal sumamente útil se le han dado múltiples usos industriales, por ejemplo, en la fabricación de pigmentos, plásticos y baterías para automóviles. De esa situación a menudo ha resultado la contaminación de amplias áreas geográficas a partir de la producción, el uso y el desecho de dichos productos. A los ministerios de salud pública les corresponde determinar las fuentes locales de exposición a plomo, ya sea de actividades industriales actuales, o bien de usos que se hicieron en el pasado, como por ejemplo, la gasolina y la pintura, respectivamente; o bien debido al depósito en terrenos y caminos de materiales contaminados por plomo. Ningún intento para solucionar este problema podrá resultar efectivo a largo plazo si no se eliminan las fuentes de exposición a dicho metal.

\section{Hábitos}

Las actividades mano-boca forman parte del comportamiento exploratorio normal de la mayoría de los niños entre los 2 y los 3 años de edad, y que desaparece en casi todos ellos. En Estados Unidos de América (EUA) este fenómeno presenta una correlación con los periodos de prevalencia pico de intoxicación por plomo en la población pediátrica. La persistencia de este hábito, cuando los niños crecen, los expone a un riesgo continuo respecto de los contaminantes ambientales. La modificación de los hábitos o conductas está indicada para aquellos niños que viven en áreas contaminadas. Al igual que el aprendizaje del control de los esfínteres, el éxito de este enfoque dependerá de la madurez del desarrollo infantil. Los reforzamientos positivos, por ejemplo los premios que se le pueden dar al niño cuando no se lleva a la boca objetos que no sean alimentos, podrían empezar a aplicarse en edades tempranas, mediante el lenguaje receptivo. Es una tarea ardua...

Otra versión de la modificación de la conducta está destinada a la persona encargada de cuidar al niño. El hecho de prestarle más atención a éste podría dar como resultado la terminación temprana de una actividad mano-boca particular. Puesto que el polvo que contiene plomo puede adherirse fácilmente a las manos, el hecho simple de morderse las uñas puede dejar algo de éste en la boca. El hábito de lavarse las manos antes de ingerir alimentos reduce el contenido que pudiera existir en las manos, pero sólo por un tiempo muy breve, apenas el necesario para comer. Por último, lavar las superficies para eliminar el polvo contaminado con plomo puede reducir el riesgo si esta actividad se lleva a cabo de manera asidua y presupone la disponibilidad de los productos de limpieza adecuados, así como de agua limpia.

\section{Nutrición}

Diversos estudios han mostrado la interacción que existe entre elementos esenciales como el calcio y el hierro. ${ }^{8}$ Se ha asociado una deficiencia de cualquiera de éstos con un incremento en la absorción y retención del plomo. La intoxicación neuronal por plomo puede resultar más grave si existe, además, una deficiencia de hierro. ${ }^{9}$ Por ello, son necesarias la ingesta adecuada de estos nutrientes y la modificación de las carencias orgánicas. En el caso de los niños de más de un año de edad la ingesta diaria de alrededor de un gramo de calcio debería satisfacer sus necesidades. Los produc- 
tos lácteos son alimentos ricos en calcio. Los antiácidos son una fuente barata de calcio si no se tolera la ingesta de productos lácteos, o bien éstos no están al alcance de la población. La carne es la fuente más segura de hierro asimilable. Puede recurrirse a los suplementos de hierro para corregir su deficiencia cuando está comprobada por niveles bajos de hemoglobina o ferritina. Actualmente se investiga si la administración de suplementos de calcio o hierro a niños intoxicados por plomo, y que no presentan deficiencias con respecto a estos metales, constituye una buena medida terapéutica.

Terapia de quelación

Definición y medicamentos. El término quelación se refiere al enlace de un metal con, al menos, dos sitios de una molécula para formar una estructura en forma de anillo. Esta definición no dice nada acerca de lo que sucede posteriormente con el metal quelado, una vez establecido el enlace. En el mejor de los casos, el tratamiento de quelación incrementará la excreción del metal tóxico con la consiguiente regresión de la intoxicación asociada con el metal. En el peor de los casos, el tratamiento quelante anulará la posibilidad de que el metal siga causando sus efectos tóxicos. Sin embargo, es de primordial importancia que la administración de una terapia quelante no ocasione un efecto pernicioso más grave que el que produce el metal mismo.

La mayor experiencia clínica tiene que ver con cuatro sustancias: edetato de calcio, succimer, BAL y penicilamina. Cada una de estas sustancias tiene diversas denominaciones, como puede apreciarse en el cuadro I. Las dosis y su posible toxicidad se indican en el cuadro II. Con excepción de la penicilamina, las dosis de las demás sustancias se calculan con base en

\begin{tabular}{|c|c|c|c|}
\hline \multicolumn{4}{|c|}{$\begin{array}{c}\text { Cuadro I } \\
\text { AgENTES PARA LA QUELACIÓN }\end{array}$} \\
\hline Producto en los EUA & Nombre genérico & Solución química & Abreviatura \\
\hline $\begin{array}{l}\text { Calcio } \\
\text { D isodio } \\
\text { Versenato }\end{array}$ & Disodio de edetato de calcio & Calcio disódico etilendiamina tetraacetato & $\begin{array}{l}\mathrm{CaNa}_{2} \\
\text { EDTA }\end{array}$ \\
\hline Bal en aceite & Dimercaprol & 2.3-dimercapto 1-propanol & BAL \\
\hline Curpimene Depen & $\begin{array}{l}\text { Penicillamine } \\
\text { Penicilamina }\end{array}$ & 3-mercapto D-valina & PCA \\
\hline Chemet & Succimer & $\begin{array}{l}\text { Meso-2.3- dimercaptosuccinic acid } \\
\text { Acido dimercaptosuccinico }\end{array}$ & DMSA \\
\hline
\end{tabular}

\begin{tabular}{|c|c|c|c|c|}
\hline \multicolumn{5}{|c|}{$\begin{array}{c}\text { Cuadro II } \\
\text { Regímenes de tratamiento }\end{array}$} \\
\hline Sustancia & Dosis inicial & Frecuencia & Vía & Duración \\
\hline $\mathrm{CaN} \mathrm{a} \mathrm{EDTA}_{2}$ & $\begin{array}{l}1000 \mathrm{mg} / \mathrm{m}^{2} / \mathrm{dí} \text { si el paciente está asinto- } \\
\text { mático; } 1500 \mathrm{mg} / \mathrm{m}^{2} / \text { día cuando existe una } \\
\text { concentración encefalopática máxima de } \\
0.5 \%\end{array}$ & $\begin{array}{l}\text { Infusión continua o bien, distribuida cada } 6 \\
012 \text { horas }\end{array}$ & $\begin{array}{l}\text { Intravenosa } 0 \\
\text { intramuscular }\end{array}$ & Cinco días \\
\hline BAL & $\begin{array}{l}300 \mathrm{mg} / \mathrm{m}^{2} / \text { día si el paciente está asintomá- } \\
\text { tico; } 500 \mathrm{mg} / \mathrm{m}^{2} / \text { día en caso de encefalo pa- } \\
\text { tía }\end{array}$ & Distribuido cada cuatro horas & $\begin{array}{l}\text { Unicamente in- } \\
\text { tramuscular }\end{array}$ & 3 a 5 días \\
\hline PCA & $\begin{array}{l}\text { Dosis inicial: } 10 \mathrm{mg} / \mathrm{kg} / \text { día. Incrementarla en } \\
\text { un lapso de dos semanas a 25-40 mg/kg/día }\end{array}$ & Distribuido cada cuatro horas & Vía oral & 12 a 20 semanas \\
\hline DMSA & $\begin{array}{l}1050 \mathrm{mg} / \mathrm{m}^{2} / \text { día durante cinco días y des- } \\
\text { pués } 700 \mathrm{mg} / \mathrm{m}^{2} / \text { día durante } 14 \text { días }\end{array}$ & $\begin{array}{l}\text { Distribuido cada ocho horas durante cinco } \\
\text { días (en el caso de los } 1050 \mathrm{mg} \text { ); distribui- } \\
\text { dos (en el caso de los } 700 \mathrm{mg} \text { ) cada } 12 \text { ho- } \\
\text { ras durante } 14 \text { días }\end{array}$ & Vía oral & Tratamiento de 19 días \\
\hline
\end{tabular}


la superficie del área corporal del niño $\left(\mathrm{m}^{2}\right)$ y no del peso (por kilogramo). La comparación de las dosis basadas en los dos métodos muestra una diferencia de hasta $25 \%$ entre ambos. Puesto que la mayoría de los estudios pediátricos se hicieron tomando en cuenta el área de la superficie del cuerpo, este es el método preferido para determinar la dosis a administrar. La dosificación por kilogramo es adecuada en el caso de los adultos.

La preferencia por una sustancia, o bien por una combinación particular de sustancias, ha variado con el tiempo. Todas pueden reducir los NPS, incrementar la excreción del plomo y reducir su toxicididad bioquímica, por lo menos durante algún tiempo. Sin embargo, en general, si se aplica un solo tipo de tratamiento se podrá eliminar sólo un porcentaje muy reducido del que se encuentra en el organismo de un niño al momento de hacer el diagnóstico. Es posible que se requieran distintos tipos de tratamientos en la terapia de quelación para poder mantener los NPS por debajo del umbral encontrado al inicio del tratamiento.

Indicaciones en la utilización de medicamentos. En los EUA existe consenso en el sentido de que la terapia de quelación está indicada en aquellos casos de niños que tengan NPS $\geq$ a $45 \mu \mathrm{g} / \mathrm{dl}^{1}{ }^{1}$ Los niños que recibieron tratamiento con succimer, y que presentaban inicialmente NPS de 20 a $44 \mu \mathrm{g} / \mathrm{dl}$, no mostraron avances cognitivos mayores que aquellos que mostraron los niños tratados con placebo. ${ }^{10}$ No obstante, es posible que existan subgrupos de niños en este rango de NPS que también hayan recibido terapia de quelación. Para poder identificar a esos niños, será necesario llevar a cabo más estudios.

Actualmente, uno de los grupos puede caracterizarse como de grandes excretores de plomo, de acuerdo con la prueba de reto de edetato de calcio. Dicha prueba permite predecir si los niños llegarán o no a excretar grandes cantidades durante la terapia de quelación. Se desconoce si los niños seleccionados para recibir terapia de quelación, de acuerdo con esta prueba, obtendrán algún beneficio a largo plazo, a pesar de que pueden demostrarse ${ }^{11}$ los efectos bioquímicos a corto plazo. Los candidatos a esta prueba deberán cumplir con las siguientes tres condiciones: tener un NPS entre 25 y $44 \mu \mathrm{g} / \mathrm{dl}$ (y no de 20 a 24), una PE >50 $\mu \mathrm{g} /$ $\mathrm{dl}$, y reservas de hierro adecuadas, con un nivel de hemoglobina de $>10.5 \mu \mathrm{g} / \mathrm{dl}$, además de un volumen corpuscular medio $(\mathrm{VCP})>73$ fl. Uno de cada tres niños que cumpla con estos requerimientos tendrá resultados positivos en la prueba de reto, por ejemplo, excretará una cantidad significativa de plomo durante

\section{Cuadro III \\ TOXICIDAD}

\begin{tabular}{|c|c|c|}
\hline Sustancia & Efectos & Frecuencia* \\
\hline $\mathrm{CaN} \mathrm{a}_{2}$ EDTA & $\begin{array}{l}\text { Gastrointestinales: pruebas de función hepática elevadas } \\
\text { Locales: inflamación del sitio donde se aplica la solución intravenosa } \\
\text { Metabólicos: hipercalcemia } \\
\text { Renales: sedimento urinario activo, proteinuria elevada } \\
\text { BUN /C reatinina } \\
\text { O rganismo: fiebre }\end{array}$ & $\begin{array}{l}\text { No es común } \\
\text { No es común } \\
\text { Dependiente de la velocidad de la aplicación } \\
\text { Rara en dosis más bajas } \\
\text { Común }\end{array}$ \\
\hline BAL & $\begin{array}{l}\text { Cardiovasculares: hipertensión, taquicardia } \\
\text { Sistema N ervioso Central: cambios en la concentración mental, psicosis } \\
\text { Gastrointestinales: náusea, vómito, dolor hepático, pruebas de función hepática elevada } \\
\text { Hematológicos: hemólisis si G } 6 P D \text { deficiente, neutropenia } \\
\text { Dermatológicos: reacciones locales en el sitio de la aplicación } \\
\text { O rgánicos: fiebre } \\
\text { N o deberá administrarse con hierro }\end{array}$ & $\begin{array}{l}\text { No es común } \\
\text { Común } \\
\text { Común } \\
\text { No es común } \\
\text { No es común } \\
\text { Común } \\
\text { No es común }\end{array}$ \\
\hline PCA & $\begin{array}{l}\text { Alérgicas: reacción con la penicilina } \\
\text { Gastrointestinales: náusea, vómito, diarrea, pruebas de función hepática elevada } \\
\text { Hematológicas: leucopenia, trombocitopenia } \\
\text { Pulmonares: tos, sibilancia } \\
\text { Renales: hematuria, preinuria, síndrome nefrótico } \\
\text { Dermatológicas: exantemas de tipo alérgico. Síndrome de Stevens-Johnson }\end{array}$ & $\begin{array}{l}\text { Común } \\
\text { Desconocida } \\
\text { Desconocida } \\
\text { Rara } \\
\text { No es común } \\
\text { Común }\end{array}$ \\
\hline DMSA & $\begin{array}{l}\text { Gastrointestinal: náusea, vómito, dolor hepático, pruebas de función hepática elevada } \\
\text { Hematológica: neutropenia, trombocitopenia } \\
\text { Dermatológica: exantemas de tipo alérgico }\end{array}$ & $\begin{array}{l}\text { No es común } \\
\text { Rara } \\
\text { No es común }\end{array}$ \\
\hline
\end{tabular}


la misma. Por otra parte, menos de 5\% de los niños tendrán resultados positivos si no cumplen con cualesquiera de los tres requerimientos: un NPS $<25$, o bien que ese nivel se encuentre en el rango 25-44 pero con una $P E<50$, o un NPS de 25 a 44 y una $P E>50$, pero con niveles de hemoglobina $<10.5$ o un $\mathrm{VCP}<73$. Los niños cuyos resultados en las pruebas de reto son positivos pueden ser sometidos a toda la serie de tratamientos que comprende la terapia de quelación.

Qué medicamento utilizar y en qué momento. Los siguientes escenarios permitirán ilustrar qué medicamentos habrán de utilizarse y en qué circunstancias.

\section{- Niño con NPS de 45 a $69 \mu \mathrm{g} / \mathrm{dl}$.}

En este caso el succimer constituye el medicamento adecuado; si el niño no lo tolera, puede ser sustituido por el edetato de calcio. En caso de no disponer de ninguno de los dos medicamentos se puede emplear, con precaución, la penacilamina.

- Niño con NPS de 70 a $100 \mu \mathrm{g} / \mathrm{dl}$.

Son dos los medicamentos indicados en este caso: el edetato de calcio y el succimer. El BAL puede sustituir al succimer, en caso de que este último no esté disponible o bien que no sea tolerado. Cuando existe evidencia de encefalopatía, por ejemplo, convulsiones vinculadas con la intoxicación por plomo, deberá recurrirse al BAL en vez del succimer. En ambos casos, el succimer o bien el BAL, deberán ser administrados cuatro horas antes de aplicar la primera dosis de edetato de calcio, ya que esta medida puede ofrecer cierta protección para el cerebro durante el tratamiento.

- Niño con NPS mayores a los $100 \mu \mathrm{g} / \mathrm{dl}$.

Es poca la información disponible acerca de los riesgos que implica administrar succimer en este rango de NPS. En el caso de los niveles aproximados a este rango, $120 \mu \mathrm{g} / \mathrm{dl}$, pueden seguirse los mismos principios que en el caso del niño con NPS de $70 \mathrm{a} 100 \mu \mathrm{g} / \mathrm{dl}$. Para los niños con niveles > a $120 \mu \mathrm{g} / \mathrm{dl}$, deberá administrarse la combinación de BAL/edetato de calcio en dosis elevadas.

- Niño con NPS de 25 a $44 \mu \mathrm{g} / \mathrm{dl}$ y resultados positivos en la prueba de reto.

Se puede aplicar cualquiera de los siguientes tres productos: edetato de calcio, succimer, o bien penicilamina. La decisión de optar por alguno de los tres es arbitraria, pues se ha observado que la eficacia de todos es de corto plazo en estos casos. Considerando la seguridad y la facilidad de uso, personalmente recomendaría la administración, en primer término, de succimer; como segunda opción, el edetato de calcio y, en tercer lugar, la penicilamina.
Atención: al utilizar estos medicamentos es necesario tomar en cuenta que todos pueden incrementar el nivel de absorción de plomo en el intestino. Por lo tanto, el tratamiento deberá aplicarse en un entorno donde no haya dicho metal.

Después. Los tratamientos de quelación pueden salvar la vida de un paciente; sin embargo, sus efectos sobre los NPS generalmente son pasajeros. Después de transcurridos algunos días o semanas a partir de terminado el tratamiento, el NPS volverá a ascender porque se trata, en principio, del plomo que se ha almacenado en hueso y que se está liberando en el torrente sanguíneo. De no ocurrir un nuevo episodio de absorción de plomo, o bien otro ciclo de tratamiento, el nivel logrará estabilizarse de cuatro a seis semanas después del tratamiento de quelación, con valores inferiores al nivel pico previo al tratamiento.

El momento indicado para verificar los NPS depende del nivel que originalmente presentaba el paciente. Se puede obtener un nivel bastante bajo un día después de haber terminado el tratamiento, y este dato permitirá documentar la efectividad de ese ciclo de tratamiento. Los pacientes con NPS mayores a los 100 $\mu \mathrm{g} / \mathrm{dl}$ antes del tratamiento, presentarán un rebote en los mismos de más de $45 \mu \mathrm{g} / \mathrm{dl}$ a los pocos días. Se recomienda dejar transcurrir un lapso de tres días entre cada serie de tratamiento, con el fin de reducir los efectos tóxicos propios de cada medicamento, y esto deberá ocurrir si la encefalopatía está bajo control. Es muy probable que los niños con NPS superiores a los $70 \mu \mathrm{g} / \mathrm{dl}$ tengan un rebote superior a los $45 \mu \mathrm{g} / \mathrm{dl}$ en unas cuantas semanas; es entonces cuando debe hacerse una nueva detección de los NPS; si éstos resultan superiores o iguales a los $45 \mu \mathrm{g} / \mathrm{dl}$, deberá reiniciarse otra serie de tratamiento. Los lineamientos para esta serie subsecuente de tratamiento son los mismos que para la primera serie.

\section{Aspectos de particular consideración}

\section{Preparación de las vísceras}

Tomando en cuenta que la típica ruta de entrada del plomo al organismo pasa por las vísceras, es prudente administrar un catártico para incrementar la motilidad intestinal y reducir el tiempo de una posterior absorción del plomo. El citrato de magnesio a menudo resulta efectivo para lograr tal propósito. Tan pronto como se inicie un movimiento en el intestino, se puede dar comienzo a la terapia de quelación. Si el paciente es sintomático, se le pueden administrar los agentes de quelación por vía parenteral, al mismo tiempo que se lleva a cabo la limpieza del intestino. 


\section{Fluidos}

En general, los agentes de quelación incrementan la excreción del plomo a través del riñón, si bien algunos también pueden aumentar la excreción a través del intestino. Por ende, cuando los NPS son elevados, habrá grandes cantidades del metal que pasarán por los riñones y parte de éste puede quedarse ahí. Con el fin de reducir la toxicidad renal vinculada con este tratamiento, que a pesar de no ser común sí se ha llegado a manifestar cuando se aplica terapia con edetato de calcio, podría resultar benéfica una mayor diuresis. Cuando la función renal es normal al inicio, y en el análisis de orina no se observa la presencia de células ni de proteínas, la liberación de fluidos para conservar una densidad específica de la orina $<1.015$ debería evitar, en principio, la toxicidad renal. En el caso de pacientes que no presentan encefalopatía, los fluidos pueden administrarse por vía oral. El cambio en la carga de volumen basal constituye un riesgo para aumentar la presión intercraneal, particularmente en los niños que presentan niveles iniciales de plomo en sangre $>100$ $\mu \mathrm{g} / \mathrm{dl}$. Para mantener un control entre estas posibles complicaciones del tratamiento, la ingesta de líquidos de mantenimiento no deberá ser riesgosa pero sí en cantidades suficientes. En caso de existir encefalopatía, o bien cuando ésta se desarrolla durante el tratamiento, es necesario restringir los fluidos para controlar la presión intracraneal.

\section{Encefalopatía}

Aun cuando no ocurre con frecuencia, la encefalopatía -convulsiones, coma- es un hallazgo premórbido. Es imperativo administrar un tratamiento con monitoreo intensivo. Los pacientes corren el riesgo de sufrir obstrucción y herniación ventricular. ${ }^{12}$ Será necesario administrarles anticonvulsivantes, además de otros agentes que permitan reducir la presión intracraneal, así como para otras causas de inflamación del cerebro. El BAL deberá administrarse en una dosis máxima de $500 \mathrm{mg} / \mathrm{m} 2 /$ día, dividida y administrada cada cuatro horas por vía intramuscular. Cuatro horas después de haberse administrado la primera dosis de BAL, puede comenzar a administrarse el edetato de calcio en una dosis de $1500 \mathrm{mg} / \mathrm{m} 2$ /día, en una infusión continua, o bien, si es necesario restringir los líquidos, se puede aplicar por vía intramuscular de manera combinada $1: 1$ con $1 \%$ de procaina, dividido en dosis para cada seis horas. Es necesario prestar mucha atención a los electrolitos séricos y a los resultados de las pruebas de funciones hepáticas; asimismo, verificar los niveles por lo menos cada tercer día. Los efectos secundarios de la medicación, incremento de la creatinina, obligarían a reducir las dosis.

En resumen, el tratamiento para el envenenamiento por plomo debe ser previsivo e incluir medidas para eliminar la exposición, evitar conductas que propicien la ingestión de este metal, disminuir las deficiencias nutricionales provocadas por su absorción, así como su retención y toxicidad, y eliminarlo del organismo mediante terapia de quelación. Todos los medicamentos utilizados en esta terapia tienen márgenes de seguridad aceptables cuando se administran con precaución y bajo una estricta supervisión. Todos pueden revertir los efectos tóxicos bioquímicos del plomo, al menos, temporalmente. Con el tratamiento aumentan los síntomas gastrointestinales. Sin embargo, sus déficits cognitivos atribuibles son de naturaleza permanente. Por ende, los principales esfuerzos deben orientarse a prevenir la intoxicación por este metal.

\section{Referencias}

1. Centers for disease Control and Prevention. Preventing lead poisoning in young children:A statement by the Centers for Disease Control.Atlanta (GA): CDC, US. Dept. of Health and Human Services, 1991.

2. Barry PSI. Distribution and storage of lead in human tissues. En: $\mathrm{N}$ riagu $\mathrm{JO}, \mathrm{Ed}$. The biogeochemistry of lead in the environment amsterdam. Elsevier/N orth Holland Biomedical Press, 1978: 97-150. 3. Rosen JF, C rocetti AF, Balbi K, Balbi J, Bailey C, Clemente I et al. Bone lead content assessed by L-line $x$-ray fluorescence in lead-exposed and non-lead exposed suburban populations in the United States. Proc N atl Acad Sci USA 1993;90: 2789-2792.

4. Markowitz ME, Rosen JF. N eed for the lead mobilization test in children with lead poisoning. J Pediatr 1991; 19:305-310.

5. Rosen JF, Markowitz ME, Bijur PE, Jenks ST,W ielopolski L, Kalef-Ezra $J A$ et al. L-line X-ray florescencse of cortical bone lead compared with the $C$ aN a2EDTA test in lead-toxic children: Public health implications. Proc N atl Acad Sci USA 1989; 86:685-689.

6.Piomelli S. Free erythrocyte porphyrins in the detection of undue absorption of lead and iron deficiency. Clin Chem 1977;23:249-259. 7. Rabinowitz MB,W etherill GW, Kopple JD. Kinetic analysis of lead metabolism in healthy humans. J Clin Invest 1976;58:260-270. 8. Markowitz ME, Rosen JF, Bijur PE. Effects of iron deficiency on lead metabolism in moderately lead toxic children. J Pediatr 1990;116: 360-364.

9. Ruff HA, Markowitz ME, Kurtzberg D, Bijur PE, Rosen JF. Relationships among blood lead levels, iron deficiency, and cognitive development in two-year-old children. Environ Health Perspect 1996;104:180-185. 10. Roan W J, D ietrich KN,W are JH, D ockery W D et al. The effect of chelation therapy with succimed on neuropsychological development in children exposed to lead. N Engl J Med 2001;344:1421-1426.

11. Morris V, Markowitz ME, Rosen JF. Serial measurements of aminolevulinic acid dehydratase in children with lead toxicity. J Pediatr 1988;112:916-919.

12. Fatal pediatric lead poisoning - N ew Hampshine, 2000. MMW R Morb Mortal W Kly Rep June 8 2001;50(22):457-459. 